\title{
Study on Antibacterial Activity against Pseudomonas Aeruginosa of Carica papaya Seed Extract
}

\author{
Nguyen Van Toan ${ }^{1,2 *}$, Nguyen ThiTruc Linh ${ }^{1}$ \\ ${ }^{1}$ School of Biotechnology, International University - Viet Nam National University in HCMC \\ ${ }^{2}$ Vietnam National University, Ho Chi Minh City, Vietnam
}

*Corresponding Author: Nguyen Van Toan, School of Biotechnology, International University - Viet Nam National University in HCMC. Vietnam.

\begin{abstract}
In this study, the antimicrobial efficacy of Carica papaya seed extract against the growth and development of Pseudomonas Aeruginosa was thoroughly investigated. Different concentrations of $0.1 \%$ to $0.2 \%$ and $0.25 \%$ of $C$. papaya seed extract cultured in two separated characteristics of medium (agar and broth) for testing the colony formation and minimum inhibitory concentration (MIC) against P. aeruginosa. The results of this study have shown that the extract of $C$. papaya seed significantly reduced the growth of $P$. aeruginosa at $0.15 \%$ and $0.21 \%$ with agar test and broth test in the ratio of $1: 4(v / v)$ respectively. On the other hand, this study gave the useful information for improving medical from extract of $C$. papaya seeds usage against the development of $P$. aeruginosa. In conclusion, the $C$. papaya seed extract could effectively prevent the growth of $P$. aeruginosa at the selectively applied concentrations.
\end{abstract}

Keywords: C. papaya seed extract, Pseudomonas aeruginosa, antimicrobial activity.

\section{INTRODUCTION}

Infectious diseases are disorders that are triggered by microorganisms, such as virus, fungi, parasite and bacterium that are directly or indirectly passed from one person to another. Infectious disease is the leading cause of death worldwide, especially in young children, particularly in low income countries[1]. Hospital-acquired infections (HAI) is an infection that is acquired in a hospital or other health care facility. HAI can be acquired in hospital, nursing home, rehabilitation facilities, outpatient clinic, diagnostic laboratory or other clinical settings. These infections consist of central line-associated bloodstream infections, catheter-associated urinary tract infections, surgical site infections, hospital-acquired pneumonia, ventilator-associated pneumonia, and Clostridium difficile infections[2]. A survey carried out in 183 United State hospitals with 11,282 patients reported that $4 \%$ of patients had at least one HAI with the most common microorganism being Clostridium difficile. Most infections were surgical site infections, pneumonia, and gastrointestinal infections. A study 2 years earlier by the same group found that $6 \%$ (51) of patients had suffered from HAI with the top $75.8 \%$ acquiring surgical site infections, urinary tract infections, pneumonia, and bloodstream infections [3]
P. aeruginosa is a type of bacteria that is found mostly in the environment, such as soil and water. It can also be found in large numbers on fresh fruits and vegetables. $P$. aeruginosa is an aerobic gram-negative bacteria and $P$. aeruginosa is specified by motile, non-spore forming rods that are oxidase positive and lactose non-fermenters. The water-soluble pigments, pyocyanin and pyoverdine, donate $P$. aeruginosa its distinctive blue-green color on solid media. Like many environmental bacteria, $P$. aeruginosa live in slime-enclosed biofilms which allow for survival and replication within human tissues and medical devices. Related to the production of a biofilm protects $P$. aeruginosa from host-produced antibodies and phagocytes contributing to antibiotic resistance of this bacteria[4]. Pseudomonas aeruginosa can cause infections in the blood, lungs (pneumonia), or other parts of the body after surgery. Infection caused by $P$. aeruginosa is common, with the load of infection in hospitalized patients. The National Nosocomial Infections Surveillance (NNIS) System records $P$. aeruginosa to be the second most common organism isolated in nosocomial pneumonia (17\% of cases), the third most common organism isolated in both urinary tract infection (UTI) and surgical site infection (11\% of cases), and the fifth most common organism isolated 
from all sites of nosocomial infection (9\% of cases). $P$. aeruginosa is an opportunistic pathogen that rarely causes disease in healthy persons. This organism is mostly considered in the differential diagnosis of a number of gramnegative infections. It is related to the nosocomial infections, often severe and lifethreatening, especially in immunocompromised hosts [4]

Due to rapid development of microbial resistance, herbal plant extract has become essential currently to screen effective, safe, cheap, and available therapeutics for their potential antimicrobial effect. According to the report of the World Health Organization, about $80 \%$ people used traditional medicine for primary health care treatment. In Asia, plants as medicine indicate long history with human involvement in the environment. Herbal medicines contain different types of novel and unique substances to treat infectious diseases[5] According to pharmaceutical studies, approaching 10 to $20 \%$ of plants are used in a positive way in health care to treat harmful diseases[6]

Carica papaya (Carica papaya L.), a kind of tropical evergreen fruit tree originated from Mexico and Central America, is mainly found distributed in South Asia. In Vietnam, it is mostly found in South Region. It is one of the herbal remedies, which has recently become a subject of research focus. It is used in traditional medicine for variety of purposes in treating infectious diseases. It is a rich source of three powerful antioxidant vitamin $\mathrm{C}$, vitamin $\mathrm{A}$ and vitamin $\mathrm{E}$; the minerals, magnesium and potassium; the B vitamin pantothenic acid and folate and fiber[7]. C. papaya seeds are edible and have spicy taste. C. papaya seeds are grinded and used as a substitute for black pepper. A small pin of C. papaya seeds over a meal is a way to add extra enzymes to the diet and improve digestive appetite.

In summary, this study has been conducted to determine as well as evaluate the possible and highly suitable concentration papaya seed extractsagainst the growth of $P$. aeruginosa. The aim of this study was to evaluate the effect of papaya seed extractsagainst the $P$. aeruginosa.

\section{Materials AND Methods}

\section{Materials}

C. papaya fruits were collected from NinhThuan province in the South-Central Coast region of Vietnam. The $C$. papaya seeds were dried overnight at $60^{\circ} \mathrm{C}$ by using drying oven (Memmert Universal Oven UN75 plus), and then kept in Desiccator for further study and analysis.

Pseudomonas aeruginosa ATCC 9027 with deep freeze format $\left(-80^{\circ} \mathrm{C}\right)$ was purchased from ATCC (American Type Culture Collection) organization, United States.

Brain Heart Infusion Broth (BHI broth) and Brain Heart Infusion Agar (BHI agar) were purchased from Ensure company, Ho Chi Minh City.

\section{Methods}

\section{Preparation of C. Papaya Seed Extract}

The well-prepared C. Papaya seeds were well ground into fine powder by using electric grinder.

To prepare the aqueous extract, $10 \mathrm{~g}$ of $\mathrm{C}$. Papaya seed powder was added to $100 \mathrm{ml}$ deionized water (ratio $1: 10 \mathrm{w} / \mathrm{v}$ ) at $90^{\circ} \mathrm{C}$ for $2-3$ hours and cool down to $50^{\circ} \mathrm{C}-55^{\circ} \mathrm{C}$. The collected mixture was filtered using standard funnel and filtering paper to collect the filtrate (aqueous phase). The filtrate was then centrifuged at the speed of 1500rpm and at room temperature for 15 minutes to remove the insoluble and debris in order to have a purified C. Papaya seed extract at a certain degree.

The following process of antimicrobial activity tests were performed with different concentrations of dilution from the total C. Papaya seed extract; computed by the formula:

$$
\mathrm{w} / \mathrm{v}(\%)=\frac{\text { mass of solute }(\mathrm{g})}{\text { volume of solution }(\mathrm{mL})} \times 100
$$

Each different final concentration was diluted using deionized water. The finalized prepared solution was autoclaved at $121^{\circ} \mathrm{C}$ and $15 \mathrm{lbs}$ pressure. After that, each solution was labeled and stored at $4^{\circ} \mathrm{C}$ for further antimicrobial tests. [8]

\section{Preparation of the Testing Microorganisms}

Pseudomonas aeruginosa ATCC 9027 with deep freeze format $\left(-80^{\circ} \mathrm{C}\right)$ was cultured in Mueller-Hinton agar (MHA) medium, then kept in an incubator (Daihan Thermostable If, Incubator, General PurposeThermostable If$50 / 105 / 155$ ) for $16-20$ hours at $37^{\circ} \mathrm{C}$ right after checking without contamination, picked randomly one colony and sub-cultured on BHI broth at $37^{\circ} \mathrm{C}$ for $16-20$ hours and used for anti- 
bacterial activity testing. To make sure only having $P$. aeruginosa appeared in $\mathrm{BHI}$ broth, the taken bacteria in BHI broth was then cultured again in MHA for $16-20$ hours at $37^{\circ} \mathrm{C}$ for gram stain and catalase test.

Before testing anti-bacterial activity, concentration of Pseudomonas aeruginosa needed to be known in order to count colony on the agar plate by making serial dilution method to make sure the number of colonies on agar plate wasn't too many or few. A plate having 30-300 colonies was chosen due to this range is considered statistically significant.

\section{Testing Antibacterial Activity}

The test based on culturing of Pseudomonas aeruginosa on the medium containing the $C$. papaya seed extracts of which followed agar test and broth test methods[ 8]

\section{Agar Plate Test}

The inoculum was cultured on BHI agar having a specific concentration of the $C$. papaya seed extract. There were three ratios as 1:4 v/v, 1:2 $\mathrm{v} / \mathrm{v}, 1: 1 \mathrm{v} / \mathrm{v}(\mathrm{mL}$ of $C$. papaya seed extract : $\mathrm{mL}$ of medium) with the name test 1 , test 2 and test 3 respectively. In each test, 12 different concentration of $C$. papaya seed extract was diluted by using this formula:

\section{$\mathrm{C} 1 \mathrm{~V} 1=\mathrm{C} 2 \mathrm{~V} 2$ Where}

$\mathrm{C} 1$ : concentration of the stock $(\%)$

V1: volume of the stock $(\mathrm{mL})$

$\mathrm{C} 2$ : concentration of the final solution $(\%)$

V2: volume of the final solution $(\mathrm{mL})$

Each different concentration repeated five times. BHI agar was autoclaved at $121^{\circ} \mathrm{C}$ for 15 minutes and let cool down to $60^{\circ} \mathrm{C}$ and then the $C$. papaya seed extract was transferred into medium and vortex well before pouring in the petri dish. $10 \mu$ l of inoculum was taken and spread on the surface of the agar with a Lshaped spreader. The agar plate was incubated at $37^{\circ} \mathrm{C}$ for $16-20$ hours. There were also several treatments which distinct in added the sample into medium [8]

Table1. Experimental design for culturing the Pseudomonas aeruginosa for each treatment

\begin{tabular}{|l|c|c|c|c|}
\hline \multirow{2}{*}{} & \multirow{2}{*}{ No. of replicated } & \multicolumn{3}{c|}{ Added materials } \\
\cline { 3 - 5 } & & Agar plate & Inoculum & C. Papaya Seed Extract \\
\hline Negative control & 1 & $\sqrt{ }$ & $\mathrm{X}$ & $\mathrm{x}$ \\
\hline Negative control 2 & 1 & $\sqrt{ }$ & $\mathrm{X}$ & $\sqrt{ }$ \\
\hline Positive control1 & 1 & $\sqrt{ }$ & $\sqrt{ }$ & $\mathrm{x}$ \\
\hline Sample & 5 & $\sqrt{ }$ & $\sqrt{ }$ & $\sqrt{ }$ \\
\hline
\end{tabular}

Table2. Experimental design for culturing Pseudomonas aeruginosa on agar plate test

\begin{tabular}{|c|c|c|}
\hline Treatment No. & Concentrate of sample & No. of replicates \\
\hline 1 & $0.25 \%$ & 5 \\
\hline 2 & $0.2 \%$ & 5 \\
\hline 3 & $0.19 \%$ & 5 \\
\hline 4 & $0.18 \%$ & 5 \\
\hline 5 & $0.17 \%$ & 5 \\
\hline 6 & $0.16 \%$ & 5 \\
\hline 7 & $0.15 \%$ & 5 \\
\hline 8 & $0.14 \%$ & 5 \\
\hline 10 & $0.13 \%$ & 5 \\
\hline 11 & $0.12 \%$ & 5 \\
\hline 12 & $0.11 \%$ & 5 \\
\hline
\end{tabular}

Colony counter (Galaxy 330's Colony Counter) was used to determine the number of colony growth and the Minimal Inhibitory Concentration of C. papaya seed extract.

Broth Test

Three different ratios as $1: \mathrm{v} / \mathrm{v}, 1: 2 \mathrm{v} / \mathrm{v}, 1: 1 \mathrm{v} / \mathrm{v}$ (C. papaya seed solution/medium) were done to determine the MIC of the extract against $P$. aeruginosa. Five replicates were applied to this test. BHI broth was autoclaved at $121^{\circ} \mathrm{C}$ for 15 Table3. Experimental design for culturing Pseudomonas aeruginosa on broth test minutes and let cool down to $60^{\circ} \mathrm{C}$, then transferred the sample to analyze into medium with a specific concentration, $10 \mu \mathrm{l}$ of inoculum was added into the solution for incubated at $37^{\circ} \mathrm{C}$ for $16-20$ hours.

\begin{tabular}{|c|c|c|}
\hline Treatment No. & Concentrate of sample & No. of replicates \\
\hline 1 & $0.25 \%$ & 5 \\
\hline 2 & $0.2 \%$ & 5 \\
\hline
\end{tabular}


Study on Antibacterial Activity against Pseudomonas Aeruginosa of Carica papaya Seed Extract

\begin{tabular}{|c|c|c|}
\hline 3 & $0.19 \%$ & 5 \\
\hline 4 & $0.18 \%$ & 5 \\
\hline 5 & $0.17 \%$ & 5 \\
\hline 6 & $0.16 \%$ & 5 \\
\hline 7 & $0.15 \%$ & 5 \\
\hline 8 & $0.14 \%$ & 5 \\
\hline 9 & $0.13 \%$ & 5 \\
\hline 10 & $0.12 \%$ & 5 \\
\hline 11 & $0.11 \%$ & 5 \\
\hline 12 & $0.1 \%$ & 5 \\
\hline
\end{tabular}

The result of this test was taken by counting the colony with "liquid serial dilution". The effect of $C$. papaya seed to the $P$. aeruginosa was determined by bacterial counting and calculated by the following formula:

$\%$ Reduction (D-value) = $\frac{[(\text { Mean CFU }) \text { control }-(\text { Mean CFU }) \text { sample }] \times 100}{(\text { Mean CFU }) \text { control }}$

$\log$

(Reduction)

$=\log _{10}[$

$\log _{10}($ Mean CFU ) sample

(Mean CFU ) control -

This method was intentionally used to define the minimum concentration of the extract solution that completely forbids the growth of visible bacteria as MIC value.

\section{Data Analysis}

All data were treated and presented as means \pm standard deviation of representatives of similar test carried out in 5 times of replication. Statistical differences in colony forming unit (CFU) were determined by student's t-test in which, P-values less than 0.05 was considered statistically significant.

\section{RESUlTS}

\section{Preparation of $C$. papaya seed extract}

After the C. papaya seed powder and deionized water were well mixed with the ratio of $1: 10$ $\mathrm{w} / \mathrm{v}$, the mixture was sonicated in the water bath (WiseClean WUC Digital Ultrasonic Cleaner) at $90^{\circ} \mathrm{C}$ for 3 hours and followed by filtering, using standard funnel, filter paper. The C. papaya seed mixture had a pungent smell and produced a layer of oil while filtering. Next, the mixture \begin{tabular}{ll|l} 
was centrifuged (Universal 320 | 320R &
\end{tabular} Centrifuge) to obtain the aqueous phase.

The obtained yield was calculated by applying the formula:

Yield of extraction $(\%)=$

\section{dissolved powder in solution $x 100$ initial biomass of powder}

The initial biomass of $C$. papaya powder $=23 \mathrm{~g}$ The insoluble powder into the solution $=21.6 \mathrm{~g}$

The dissolved powder into the solution $=1.4 \mathrm{~g}$

Thus, the yield of extraction is $6.09 \%$

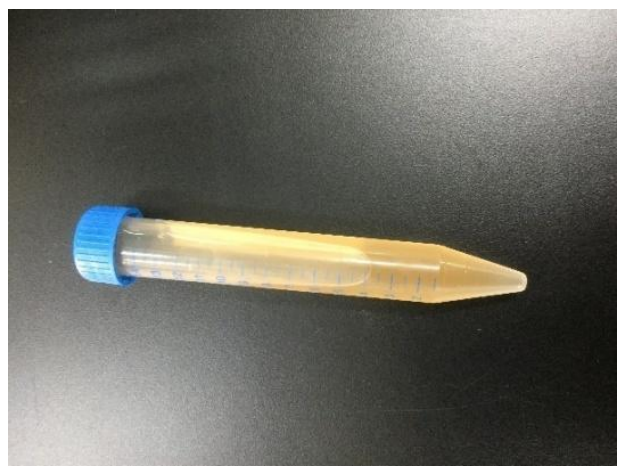

Figure1. The final solution of C. papaya seed extract

The $C$. papaya seed extract had a pale-yellow color after the extraction. From the dried materials of $C$. payaya seeds $(23 \mathrm{~g})$, by extraction method $21.6 \mathrm{~g}$ the insoluble powder was obtained. After applying the yield of extraction process formula, the extraction yield of $C$. papaya seed extract was $6.09 \%$.
The total $C$. papaya seed extract was diluted into different concentrations and used in the process of antimicrobial activity tests, computed using the formula:

$$
\begin{aligned}
\mathrm{w} / \mathrm{v} \% & =\frac{\text { mass of solute }(\mathrm{g}) \times 100}{\text { volume of solution }(\mathrm{mL})} \\
\mathrm{w} / \mathrm{v} \% & =\frac{1.4(\mathrm{~g}) \times 100}{140(\mathrm{~mL})}=1 \%
\end{aligned}
$$


The stock solution had the concentration as $1 \%$ (w/v\%). From the stock solution, 12 concentration of $C$. papaya seed were made $(0.1 \%, 0.11 \%, 0.12 \%, 0.13 \%, 0.14 \%, 0.15 \%$, $0.16 \%, 0.17 \%, 0.18 \%, 0.19 \%, 0.2 \%, 0.25 \%$ ) in order to test antimicrobial activity.

\section{Preparation of Microorganisms}

Identification of the Cultured Streptococcus mutans

\section{Identification of the Cultured $P$. aeruginosa}

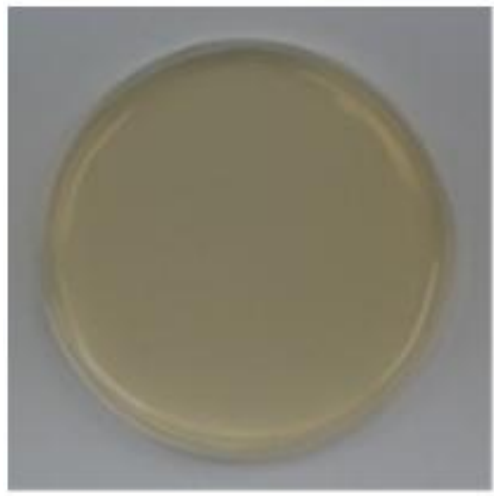

Negative Control
$P$. aeruginosa is a member's belongings to Pseudomonadaceae family (a member of the Gammaproteobacteria). On the MHA plate, the colony formation has exposed green color due to Pyocyanin produced and diffused through the medium. The colony of $P$. aeruginosa was formed on MHA and BHI agar after incubating at $37^{\circ} \mathrm{C}$ for $16-20$ hours as completely opposed to the negative control was indicated in the Figure 2and Figure 3respectively.

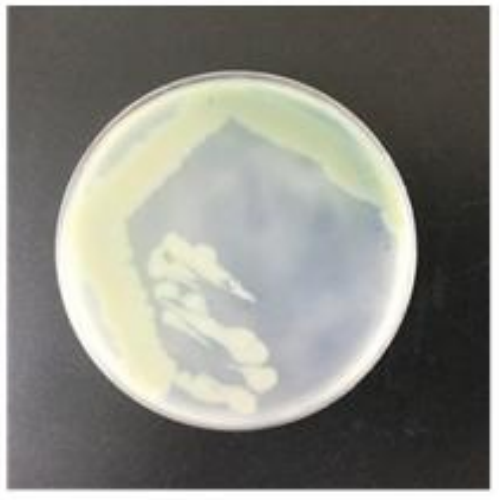

P. aeruginosa grow on $\mathrm{MHA}$

Figure2. Pseudomonas aeruginosa grown on MHA

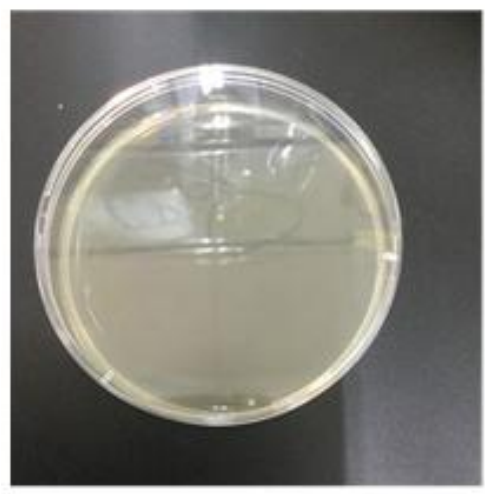

Negative Control

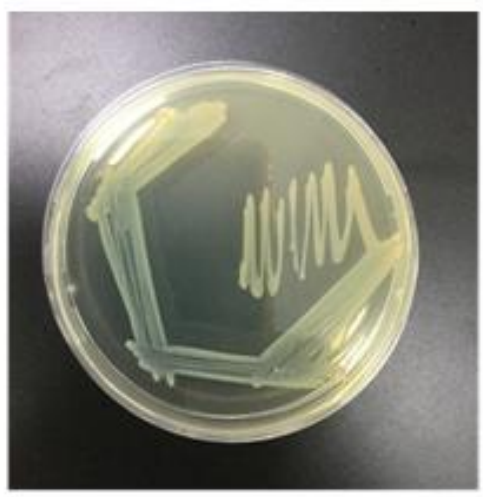

P. aeruginosa grow on $\mathrm{BHI}$ agar

Figure3. Pseudomonas aeruginosa grown on BHI agar

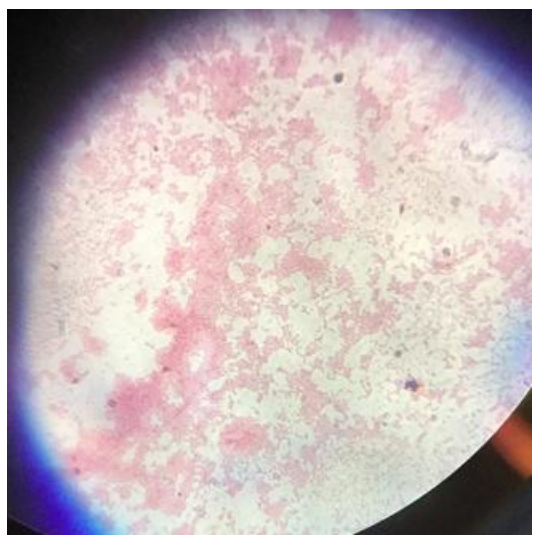

Figure4. Gram stain of P. aeruginosa 


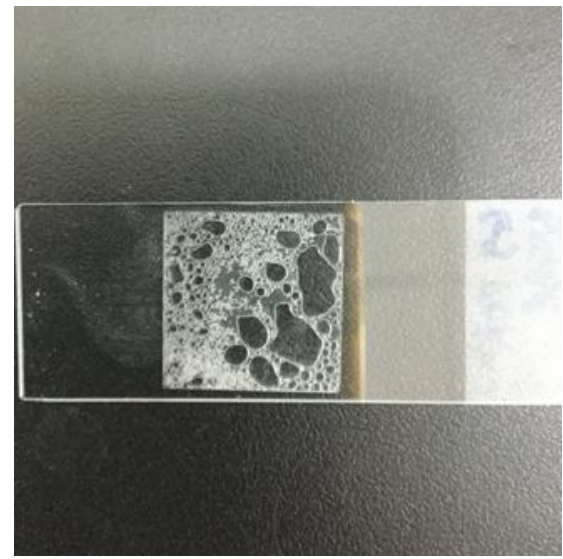

Figure5. Catalase test of $P$. aeruginosa

Serial Dilution Method on $P$. aeruginosa

Picked up colonies on BHI agar plate and incubated in BHI broth overnight at $37^{\circ} \mathrm{C}$. Diluted the suspension by taking $1 \mathrm{~mL}$ from the stock solution to $9 \mathrm{~mL}$ of BHI broth. After first tube, each tube was the dilution $(1: 9)$ of the previous tube. Repeated this step until 6 test tubes with different concentration was done, then measured OD for each tube. $10 \mu \mathrm{l}$ of each test tube was spread on BHI agar plate and incubated overnight at $37^{\circ} \mathrm{C}$.

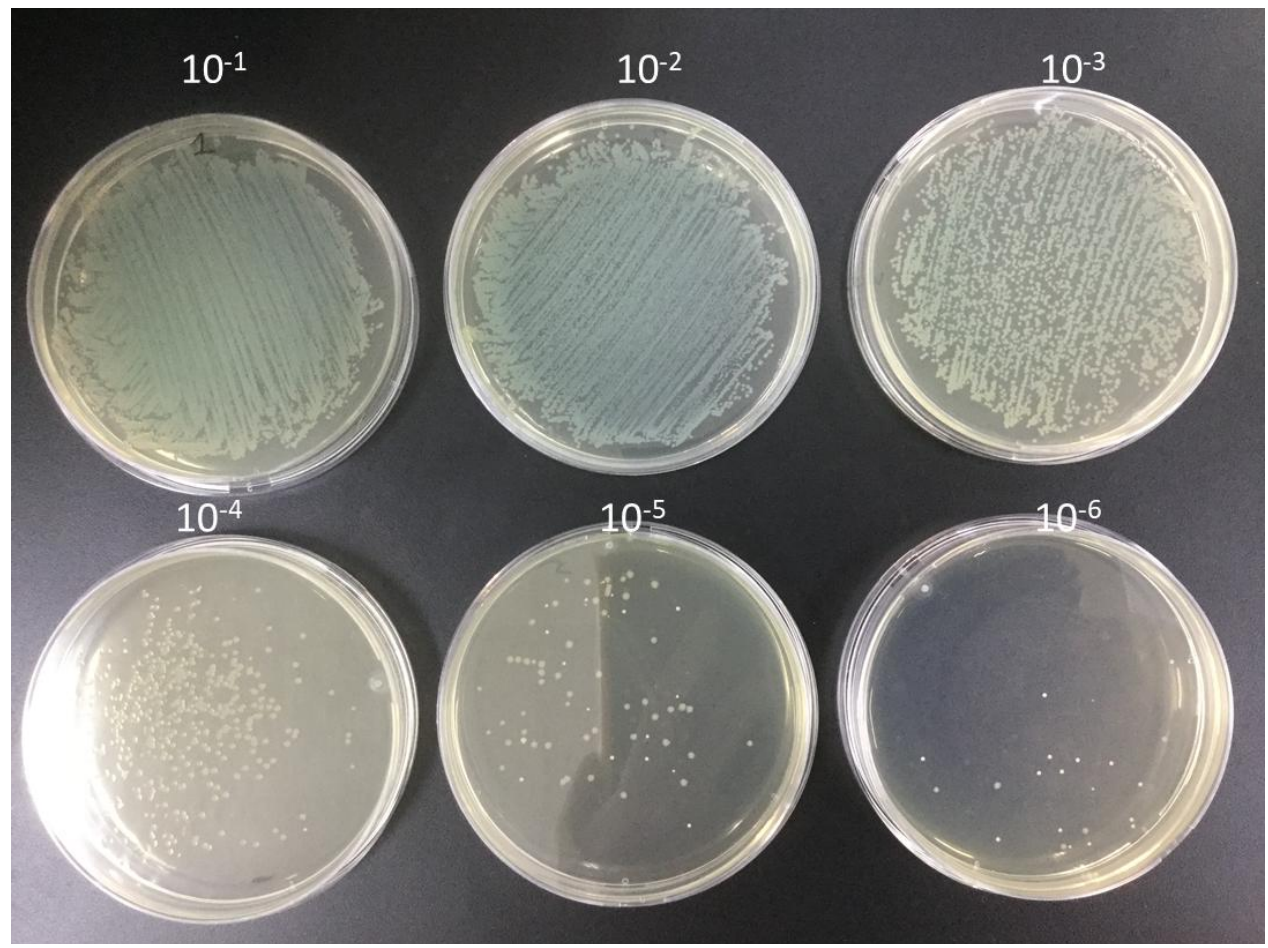

Figure6. Serial dilution of P. aeruginosa performed on BHI agar

Figure 6 presented the $10^{-4}(\mathrm{CFU} / \mathrm{mL})$ was relevant with 184 colonies formed on $\mathrm{BHI}$ agar. According to the result shown on Figure 3.3.2.1, the stock solution colony formation unit was $2 \times 10^{6}$. The OD recorded on $10^{-4}(\mathrm{CFU} / \mathrm{mL})$ was at 0.008 .

\section{Determination of Antimicrobial Activity}

\section{Agar Plate Test}

In the agar plate test, the experiment was carried out by using two controls (positive and negative controls) and three testing samples, including ratios as $1: 4 \mathrm{v} / \mathrm{v}, 1: 2 \mathrm{v} / \mathrm{v}$ and $1: 1 \mathrm{v} / \mathrm{v}$ (mL of $C$. papaya seed extract : $\mathrm{mL}$ of medium) as well as with twelve different concentrations of $C$. payaya seed extraction $(0.1 \%, 0.11 \%, 0.12 \%$, $0.13 \%, 0.14 \%, 0.15 \%, 0.16 \%, 0.17 \%, 0.18 \%$, $0.19 \%, 0.2 \%$ and $0.25 \%(\mathrm{w} / \mathrm{v}))$ for each the ratios, with name test 1 , test 2 , test 3 respectively. The experiment was repeated five times with each ratio and concentration. After adding $C$. papaya seed solutions and incubating at $37^{\circ} \mathrm{C}$ for $16-20$ hours, the data will be recorded. 
Study on Antibacterial Activity against Pseudomonas Aeruginosa of Carica papaya Seed Extract

Table4. The antimicrobial activity result of $C$. papaya seed extract testing against P. aeruginosa after incubation at $37^{\circ} \mathrm{C}$ for 16-20 hours on BHI agar plate

\begin{tabular}{|c|c|c|c|c|c|c|}
\hline No. of test & & $\begin{array}{c}\text { Concentration of } C \text {. } \\
\text { papaya seed extract } \\
(\% \mathrm{w} / \mathrm{v})\end{array}$ & $\begin{array}{c}\text { Mean of } P \text {. } \\
\text { aeruginosa colony } \\
\text { formation per } \\
\text { plate }\end{array}$ & $\begin{array}{l}\text { Standard } \\
\text { deviation }\end{array}$ & \begin{tabular}{|c|} 
Reduction of $P$. \\
aeruginosa colony \\
formation per \\
plate $(\%)$
\end{tabular} & Log reduction \\
\hline \multirow[t]{6}{*}{ Test $1(1: 4)$} & Control & & 207.5 & 81.51 & & \\
\hline & & $0.1 \%$ & 75.8 & 20.82 & 63.47 & 0.44 \\
\hline & & $0.11 \%$ & 58.2 & 16.58 & 71.95 & 0.55 \\
\hline & & $0.12 \%$ & 44.4 & 6.34 & 78.60 & 0.67 \\
\hline & & $0.13 \%$ & 5 & 1.26 & 97.60 & 1.62 \\
\hline & & $0.14 \%$ & 1.6 & 1.35 & 99.23 & 2.11 \\
\hline \multirow[t]{4}{*}{ Test 2 (1:2) } & Control & & 133.5 & 68.86 & & \\
\hline & & $0.1 \%$ & 30.8 & 5.07 & 76.93 & 0.64 \\
\hline & & $0.11 \%$ & 15.4 & 3.9 & 88.47 & 0.94 \\
\hline & & $0.12 \%$ & 0.4 & 0.48 & 99.70 & 2.52 \\
\hline Test $3(1: 1)$ & Control & & 227.6 & 67.22 & & \\
\hline
\end{tabular}

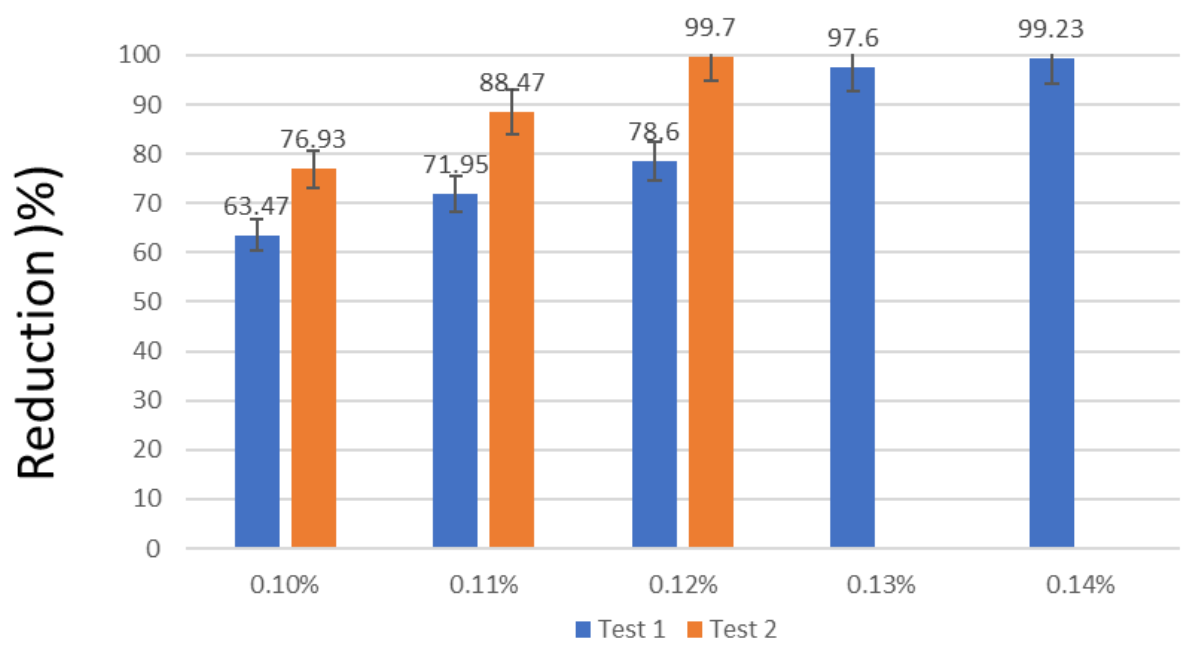

Figure7. The percentage of bacterial reduction (\%) at different concentration of $C$. papaya seed extract with different ratios in agar media

\section{Broth Test}

The broth experiment was conducted with three control samples and three ratios testing samples as $1: 4 \mathrm{v} / \mathrm{v}, 1: 2 \mathrm{v} / \mathrm{v}$ and $1: 1 \mathrm{v} / \mathrm{v}$ ( $\mathrm{ml}$ of medium: $\mathrm{ml}$ of $C$. papaya seed extract) with different concentrations of the extract and each experiment was repeated five times. The turbidity of the solution was used to test the bacterial growth after incubating for 16-20 hours, and dilution method was used to count colony from a broth medium that sub-cultured on agar plates. Taking $1 \mathrm{~mL}$ of the inoculum suspension mixed with $9 \mathrm{~mL}$ of sterile distilled water. From this suspension, 12-fold dilution factor was made and $0.1 \mathrm{~mL}$ of final dilution was cultured on agar plated at $37^{\circ} \mathrm{C}$ for $16-20$ hours.

Table5. The antimicrobial activity result of $C$. papaya seed extract testing against $P$. aeruginosa after subculture and incubation at $37^{\circ} \mathrm{C}$ for 16-20 hours on BHI agar plate from broth test pieces.

\begin{tabular}{|c|c|c|c|c|c|c|}
\hline No. of test & & $\begin{array}{c}\text { Concentration of } C . \\
\text { papaya seed extract } \\
(\% \mathrm{w} / \mathrm{v})\end{array}$ & $\begin{array}{c}\text { Mean of } P . \\
\text { aeruginosa colony } \\
\text { formation per } \\
\text { plate }\end{array}$ & $\begin{array}{c}\text { Standard } \\
\text { deviation }\end{array}$ & $\begin{array}{c}\text { Reduction of } P . \\
\text { aeruginosa colony } \\
\text { formation per } \\
\text { plate }(\%)\end{array}$ & Log reduction \\
\hline \multirow{3}{*}{ Test 1 (1:4) } & Control & & 207.5 & 81.51 & & \\
\cline { 2 - 7 } & & $0.12 \%$ & 142.8 & 36.18 & 31.2 & 0.16 \\
\cline { 2 - 7 } & & $0.13 \%$ & 128.6 & 24.71 & 38 & 0.2 \\
\cline { 2 - 7 } & & $0.14 \%$ & 103.4 & 27.6 & 50 & 0.3 \\
\cline { 2 - 7 } & & $0.15 \%$ & 101.8 & 38.2 & 51 & 0.309 \\
\cline { 2 - 7 } & $0.16 \%$ & 84.4 & 7.3 & 59.33 & 0.4 \\
\hline
\end{tabular}


Study on Antibacterial Activity against Pseudomonas Aeruginosa of Carica papaya Seed Extract

\begin{tabular}{|c|c|c|c|c|c|c|}
\hline & & $0.17 \%$ & 83.2 & 26.16 & 60 & 0.4 \\
\hline & & $0.18 \%$ & 45.2 & 16.02 & 78.22 & 0.66 \\
\hline & & $0.19 \%$ & 10.8 & 8.3 & 94.8 & 1.28 \\
\hline & & $0.2 \%$ & 0.6 & 0.8 & 99.77 & 2.54 \\
\hline \multirow{7}{*}{ Test $2(1: 2)$} & Control & & 133.5 & & & \\
\hline & & $0.13 \%$ & 128.2 & 17.37 & 4.12 & 0.02 \\
\hline & & $0.14 \%$ & 113.4 & 22.00 & 15.06 & 0.07 \\
\hline & & $0.15 \%$ & 73.8 & 18.69 & 64.44 & 0.25 \\
\hline & & $0.16 \%$ & 36.6 & 16.95 & 72.59 & 0.56 \\
\hline & & $0.17 \%$ & 20.8 & 10.32 & 84.42 & 0.8 \\
\hline & & $0.18 \%$ & 0.8 & 1.16 & 99.4 & 2.22 \\
\hline Test $3(1: 1)$ & Control & & 227.6 & 67.22 & & \\
\hline
\end{tabular}

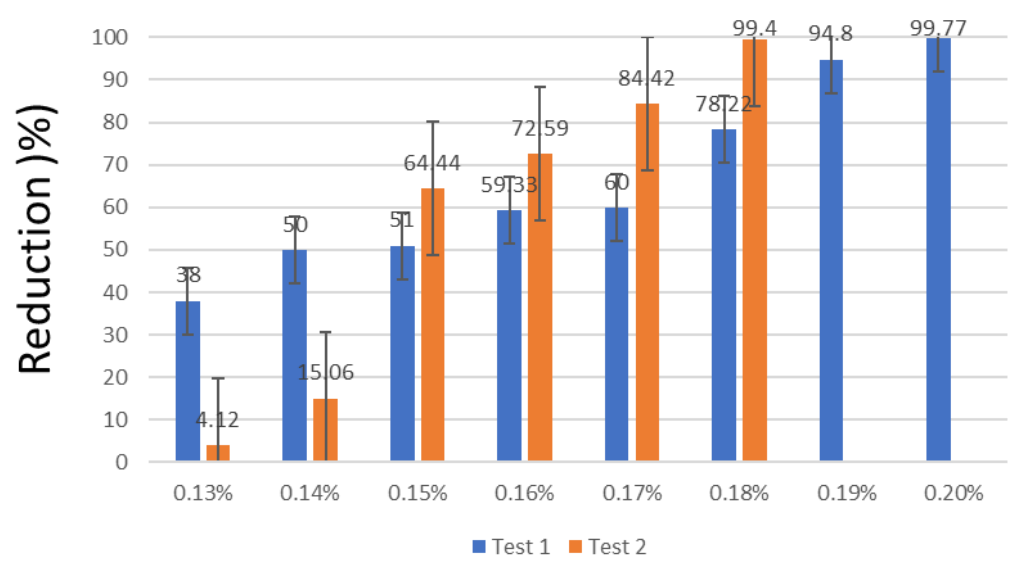

Figure8. The percentage of bacterial reduction (\%) at different concentration of $C$. papaya seed extract with different ratios in broth media

\section{DISCUSSION}

Carica papaya seed extracts inhibited majority of tested microorganisms[9]

Pseudomonas aeruginosa ( $P$. aeruginosa) is a highly drug resistant and opportunistic pathogen. Due to the permeability barrier in the outer membrane it is naturally resistant to many antibiotics. Infections caused by P. aeruginosa are increasing both in hospitals and in general community and it has been reported as one of the principal causes of nosocomial pathogen, particularly among immuno-compromised patients [10]. People with cystic fibrosis, burn victims, individuals with cancer, and persons infected with HIV are particularly at risk of disease resulting from Pseudomonas aeruginosa. Unlike many environmental bacteria, Pseudomonas aeruginosa has a remarkable capacity to cause disease in susceptible hosts [11 ].

From the all presented results, it can easily be seen that the identification tests were appropriately conducted and that was why the all collected data had shown a clear picture of inhibition of Carica papaya seed extract against the growth of $P$. aeruginosa at certain degrees.
For the testing with agar plate, the bacterial reduction of different concentration as $0.1 \%$ and $0.11 \%$ of test 1 and 2 were significant differences. Comparisons of $P$. aeruginosa reduction showed no significant differences between the $0.13 \%, 0.14 \%$ in test 1 of $C$. papaya seed extract concentrations. Because of the differences among the other concentrations of the extract between $0.1 \%$ to $0.13 \%$ in test 1 and $0.1 \%$ to $0.12 \%$ in test 2 were significant $(\mathrm{p}<0.05)$ and from $0.14 \%$ to $0.2 \%$ and $0.25 \%$ was no significant difference. When the concentration of sample increasing, the probability of colony formation on agar plates would be a clear reduction in order of $0.1 \%$, and $0.11 \%$ concentrations in test 1 , test 2 , and approximate or no colony from the concentration of $0.13 \%$ to $0.2 \%$ and $0.25 \%$ in three tests. So, the proportion of bacterial reduction underwent a rise as well as the increasing concentration of $C$. papaya seed extract. As a result, the increased amount of $C$. papaya seed extract in the medium inhibited the growth of $P$. aeruginosa at a wider range.

In the case of broth testing, as shown in the Figure 7 and Figure 8, At the concentration of $0.19 \%$ in test $1,0.18 \%$ in test 2 , the $C$. papaya 
seed extract started their antimicrobial activity with the percentage of $P$. aeruginosa reduction was higher than $90 \%$ due to the evenly low colony formation.

Also, from the Table 5, the percentage of bacterial reduction performing in broth medium, after the broth culture was put on agar plate, and then incubated in 24 hours for counting the colony formation units as CFU. The CFU was calculated for the reduction's proportion to determine whether sample had antimicrobial activity or not. It was finally found that the growth of $P$. aeruginosa was about to be inhibited by the C. Papaya seed extract for at least 24 hours at the concentration of $0.20 \%$ with one-fourth per total solution.

\section{CONCLUSIONS}

In this study, the effects of $C$. papaya seed extract against the growth of $P$. aeruginosa were successful investigated. The extracted concentration of $C$. papaya was established that at least $0.14 \%(\mathrm{w} / \mathrm{v})$ of one-fourth per total medium preparation reduced the proportion of $P$. aeruginosa over $95 \%$. The extracted concentration of the C. papaya seeds at $0.20 \%$ of the most suitable concentration, which showed a minimal and most effective on the inhibition of $P$. aeruginosa.

Compare to other studies, water extract of $C$. papaya seed had lower effect than ethanol extract.

\section{REFERENCE}

[1] Baylor College of Medicine. 2020. Introduction to Infectious Diseases. [online] Available at: $<$ https://www.bcm.edu/departments/molecularvirology-and-microbiology/emerging-infections -and-biodefense/introduction-to-infectiousdiseases> [Accessed $17^{\text {th }}$ September 2020].
[2] Alberto F. Monegro, VijayadershanMuppidi, Hariharan Regunath.Hospital Acquired Infections, University of Missouri, August 11, 2020.

[3] Mainul Haque, Massimo Sartelli, Judy McKimm, and Muhamad Abu Bakar. Health care-associated infections - an overview, Infect Drug Resist. 2018; 11: 2321-2333. 15th November 2018.

[4] Shigeki Fujitani, M.D., Kathryn S. Moffett, M.D., Victor L. Yu, M.D. Pseudomonas aeruginosa. Accessed 17th September 2020, http://www.antimicrobe.org/new/b112.asp\#r59

[5] V. Duraipandiyan, M. Ayyanar, and S. Ignacimuthu, "Antimicrobial activity of some ethnomedicinal plants used by Paliyar tribe from Tamil Nadu, India," BMC Complementary and Alternative Medicine, vol. 6, no. 1, p. 35, 2006.

[6] M. Naczk and F. Shahidi, "Phenolics in cereals, fruits and vegetables: occurrence, extraction and analysis," Journal of Pharmaceutical and Biomedical Analysis, vol. 41, no. 5, pp. 15231542, 2006.

[7] Aravind. G, Debjit Bhowmik, Duraivel. S, Harish. G. Traditional and Medicinal Uses of Carica papaya, Volume 1, 2013.

[8] Toan, V.N., \& Dung, T.K.N. (2018). Study of antibacterial activity against Streptococcus mutans of C. Papaya seed extract. International Journal of Research Studies in Medical and Health Sciences, 3(9), Pages 6-17

[9] Kaibing Zhou et al, " Antioxidant Activity of Papaya Seed Extracts", Molecules 2011, 16, 6179-6192; doi:10.3390/molecules16086179

[10] Lee, Y.C., Ahn, B.J, Jin, J.S., et al., Molecular characterization of Pseudomonas aeruginosaisolatesresistant to all antimicrobial agents, but susceptible to colistin, in Daegu. Korea. J Microbiol.(2007)

[11] Tanya, S., Daniel Y., Pseudomonas aeruginosa - a phenomenon of bacterialresistance., Journal of Médical Microbiology (2009)

Citation: Nguyen Van Toan \& Nguyen ThiTruc Linh, "Study on Antibacterial Activity against Pseudomonas Aeruginosa of Carica papaya Seed Extract”, International Journal of Research Studies in Medical and Health Sciences. 2021; 6(1): 01-09. DOI: https://doi.org/10.22259/ijrsmhs.0601001

Copyright: (C) 2021 Nguyen Van Toan \& Nguyen ThiTruc Linh, This is an open-access article distributed under the terms of the Creative Commons Attribution License, which permits unrestricted use, distribution, and reproduction in any medium, provided the original author and source are credited. 